\title{
Levels of serum IgG subclasses in patients with liver disease: A retrospective study
}

\author{
WEI ZHENG ${ }^{1}$, FEIFEI JIANG ${ }^{1}$, JING SHAN ${ }^{2}$, YING WANG ${ }^{1}$, YONGMEI JIA ${ }^{1}$, \\ QIUYAN GUO ${ }^{1}$, JINLI LOU ${ }^{1}$ and YAN ZHAO ${ }^{1}$ \\ ${ }^{1}$ Clinical Laboratory Center and ${ }^{2}$ Department of Hepatology and Immunology, \\ Beijing YouAn Hospital, Capital Medical University, Beijing 100069, P.R. China
}

Received February 17, 2020; Accepted September 15, 2020

DOI: $10.3892 / \mathrm{etm} .2020 .9476$

\begin{abstract}
Viral and alcoholic liver disease, drug induced liver disease (DILD), primary biliary cirrhosis (PBC) and autoimmune hepatitis (AIH) are among the most common liver diseases observed in clinical practice. These diseases lack unique clinical characteristics at the beginning of pathogenesis, which renders specific diagnosis difficult. Immunoglobulin $\mathrm{G}(\mathrm{IgG})$ subclasses are the main isoform of antibodies that can be found in the serum that serve important protective roles in immunity. The present study aimed to investigate the serum IgG subclass distribution in patients with the five common liver diseases aforementioned. The present study retrospectively recorded and analyzed the serum IgG subclass levels of different patients, who were grouped according to their clinical diagnosis. Serum IgG subclass levels were measured using immunonephelometric assays. IgG3 levels were found to be significantly increased whereas IgG4 levels were significantly decreased in patients with PBC. In patients with $\mathrm{AIH}, \mathrm{IgG1}$ levels were significantly increased. By contrast, IgG1/IgG level ratios in patients with viral liver disease were significantly increased. No clear pattern in the distribution characteristics of IgG subclasses could be observed in cohorts with alcoholic liver disease and DILD in the present study. Additionally, model for end-stage liver disease scores regarding IgG1 in patients with AIH shared a synergistic relationship. Anti-mitochondrial antibody subtype
\end{abstract}

Correspondence to: Dr Yan Zhao, Clinical Laboratory Center, Beijing YouAn Hospital, Capital Medical University, 8 Xitoutiao, Youanmenwai, Fengtai, Beijing 100069, P.R. China

E-mail: 18911380390@163.com

Abbreviations: DILD, drug-induced liver disease; PBC, primary biliary cirrhosis; AIH, autoimmune hepatitis; $\mathrm{HCs}$, healthy controls; IgG, immunoglobulin G; IgG4-RD, IgG4-related disease; MELD, model for end-stage liver disease

Key words: IgG, viral liver disease, alcoholic liver disease, druginduced liver disease, primary biliary cirrhosis, autoimmune hepatitis
M2 (AMA-M2) and IgG3 in patients with PBC demonstrated a synergistic relationship. These results suggested that $\mathrm{IgG}$ subclasses may be used as biomarkers to further the understanding of liver disease, which could allow for early diagnosis.

\section{Introduction}

Chronic liver disease and its associated complications are responsible for $>2$ million deaths per year worldwide (1-3). Viral and alcoholic liver disease, drug induced liver disease (DILD), primary biliary cirrhosis (PBC) and autoimmune hepatitis $(\mathrm{AIH})$ are among the most common types of liver diseases observed in clinical practice $(4,5)$. However, these diseases lack unique clinical characteristics, making their diagnoses difficult to distinguish. Generally, the causes of viral liver disease, alcoholic liver disease and DILD are clearly defined. However, PBC and AIH are autoimmune diseases that have no known clear causes. Certain autoimmune antibodies, including anti-mitochondrial antibodies (AMAs) and anti-nuclear antibodies (ANAs), have been reported to be important for the diagnosis of autoimmune diseases (6). A disrupted balance between pathogen recognition and the innate and adaptive immune systems is a common cause of liver disease progression (7).

Immunoglobulin $\mathrm{G}(\mathrm{IgG})$ is a major antibody isotype in the blood that protects the body against pathogenic infection. The IgG family is comprised of $\mathrm{IgG} 1, \mathrm{IgG} 2$, IgG3 and IgG4 (8). Differences in the structural composition of the IgG subclasses determines their function, which include antigen binding, immune complex formation, complement activation, triggering of effector cells, half-life and placental transport (9). IgG1 is the most abundant subclass in the serum, whilst IgG3 has the shortest half-life (7 days) (10). In addition, responses to different antigens will lead to marked skewing towards specific IgG antibody subclasses (11). Although deficiencies in selective subclasses are not usually harmful, they may lead to enhanced susceptibility towards specific classes of pathogens. However, this condition is rarely observed (12). IgG subclasses contribute to the immunopathogenesis of liver diseases by regulating immunoglobulin $\mathrm{Fc} \gamma$ receptor $(\mathrm{FcgR})$ and complement interactions (13). Radioimmunodiffusion or ELISA assays can be used to measure the levels of specific serum IgG subclasses but are not particularly accurate or convenient (14). 
Immunonephelometric assays are therefore the preferred method for this application (15).

IgG4-related disease (IgG4-RD) is a fibroinflammatory, immune system-mediated systemic disease that was reported for the first time in 2014 in Japan (16). IgG4-RD is described as a novel clinical entity of unknown origin that involves multiple organs (17). The current diagnostic approach for IgG4-RD includes serum IgG4 levels $>135 \mathrm{mg} / \mathrm{dl}$ (18). Other diagnostic criteria include IgG4-related tests, imaging and typical histopathological examination (19). However, accurate diagnosis of IgG4-RD is challenging, since IgG4-RD can be easily misdiagnosed as autoimmune pancreatitis, inflammatory pseudotumour and Küttner's tumor (20). IgG4-related hepatobiliary diseases include IgG4-related sclerosing cholangitis and IgG4-related hepatopathy (21).

Previous studies have focused on the relationship between the IgG subclasses and diseases that affect the body's immunity, including the significant increase of IgG1 and IgG3 in patients with rheumatoid arthritis (22), IgG subclass determination in renal disease progression (23) and distinguishing patients with human immunodeficiency virus infections who exhibit varying degrees of infection (24). However, few prior studies have previously analyzed serum IgG subclass levels fully in clinically common liver diseases and limited data are available regarding IgG4 levels in larger patient cohorts for diseases other than IgG4-RD (25-27). Both liver diseases and IgG4-RD exhibit changes in IgG4 levels (28). Therefore, to further understanding, provide an early diagnosis strategy of different liver diseases and evaluation of $\mathrm{IgG} 4$ levels in patients with non-IgG4-RD, the present study analyzed serum IgG subclass levels in patients with five common liver diseases.

\section{Materials and methods}

Patients. Clinical information from 32 patients with viral liver disease, 26 with alcoholic liver disease, 39 with DILD, 28 with PBC, 29 with AIH and 30 healthy controls (HCs) was recorded at the Clinical Laboratory Center, Beijing YouAn Hospital, Capital Medical University (Beijing, China) between May 2018 and March 2019. Patients with other types of liver or autoimmune diseases, including acute and chronic fatty liver disease, metabolic liver diseases, rheumatal-immune diseases and systemic lupus erythematosus, were excluded from the present study. The patients recruited into the present study only had diseases affecting the liver. All patients with viral liver disease met the guidelines of Prevention and Treatment for Chronic hepatitis B (29) and C (30). Patients with alcoholic liver disease met the 2010 American Association for the Study of Liver Diseases diagnosis (AALSD) (31). Those with DILD met the diagnostic criteria of the Roussel Uclaf Causal Relationship Assessment Method (RUCAM), with a RUCAM causality scale of $>6$ points (32). Patients with PBC met the 2018 AALSD PBC diagnosis guidelines (33). Patients with AIH met the 2015 Chinese Consensus on the Diagnosis and Management of Autoimmune Hepatitis criteria (34). The laboratory test results, including alanine transaminase (ALT), aspartate aminotransferase (AST), $\gamma$-glutamyl transferase (GGT), alkaline phosphatase (ALP), bilirubin and albumin of $\mathrm{HCs}$, were found to lie within the normal range. All baseline information of the patients recruited into the present study, including age, sex distribution and laboratory parameters, are listed in Table I. The present study was approved by the Ethics Committee of Beijing YouAn Hospital, Capital Medical University (Beijing, China).

IgG and other indices measured. Baseline blood samples were collected from all participants and all samples were processed at the Clinical Laboratory Center of Beijing YouAn hospital, Capital Medical University. Serum IgG subclass levels were measured using immunonephelometric assays using molecular biology kits (N Latex IgG1, cat. no. OQXI; N Latex IgG2, cat. no. OQXK; N Latex IgG3, cat. no. OPAV; $\mathrm{N}$ Latex IgG4, cat. no. OPAU and N Supplementary Reagent/Precipitation; all Siemens Healthineers) in Siemens BNII automatic protein analyzer (Siemens Healthineers) according to the manufacturer's protocols. IgG levels were determined regardless of disease stage. The normal ranges of adult IgG subclasses were defined as follows: i) IgG1, 4.05-10.11 g/l; ii) IgG2, 1.69-7.86 g/l; iii) IgG3, 0.11-0.85 g/l; and iv) $\mathrm{IgG} 4,0.03-2.01 \mathrm{~g} / 1$ (normal ranges derived from $\mathrm{IgG}$ subclass kit instructions). Serum IgG levels were defined as the sum of $\operatorname{IgG1}, \operatorname{IgG} 2$, IgG3 and IgG4.

IgM (cat. no. OSAT) and IgA (cat. no. OSAR) levels were also determined by electrochemiluminescence using a Modular E170 analyzer (Roche Diagnostics). Anti-mitochondrial antibody (AMA), anti-nuclear antibody (ANA) and anti-smooth muscle antibody (ASMA) levels were measured using an indirect immunofluorescence assay (cat. no. FA1510-1; EuroImmun AG; PerkinElmer, Inc.). AMA type 2 (AMA-M2) levels were measured using ELISA (cat. no. EA1590-9601-8G; EuroImmun AG; PerkinElmer, Inc.). Autoantibodies titers $\geq 1: 100$ were considered positive and an absorbance value of AMA-M2 $\geq 25 \mathrm{RU} / \mathrm{ml}$ was also considered positive.

The model for end-stage liver disease (MELD) score used in the present study was obtained from the United Network for Organ Sharing (UNOS) for the prioritization of transplant organs, which was calculated using the following formula (35): $\mathrm{MELD}=3.8 \mathrm{x}\{\mathrm{Ln}[$ serum bilirubin $(\mathrm{mg} / \mathrm{dl})]\}+11.2 \mathrm{x}\{\mathrm{Ln}$ [international normalized ratio (INR) $]\}+9.6 x\{\operatorname{Ln}$ [serum creatinine $(\mathrm{mg} / \mathrm{dl})]\}+6.4 \mathrm{x}$ (constant for liver disease etiology). Ln was the natural logarithm with base e. If disease etiology was biliary or alcoholic, constant for liver disease etiology was considered to be 0 whereas other etiologies, such as viral hepatitis, were set to 1 . A MELD score $>6$ was considered to be abnormal.

Statistical analysis. Normally distributed numerical variables were assessed using Q-Q plot graphical methods and were presented as the mean \pm SD. Data were instead presented as the median (interquartile range, 25 th and 75 th percentile) when numerical variables were not normally distributed. Categorical variables were presented as numbers and percentages. One-way ANOVA and Kruskal-Wallis tests were performed to compare IgG subclass levels. Multiple comparison between the groups was performed using Scheffe's post hoc test. Statistical differences between two groups were analyzed using Student's t-test or Mann-Whitney test. $\chi^{2}$ test was applied to analyze the percentages of elevated IgG subclasses. Additionally, Pearson's correlation coefficient was used to evaluate the relationship between the levels of two subclasses of antibodies. $\mathrm{P}<0.05$ 
was considered to indicate a statistically significant difference. SPSS version 22.0 (IBM Corp.) and GraphPad Prism version 7.0 for Windows (GraphPad Software, Inc.) were used for data analysis. All experiments were repeated three times and there were three replicates for each experiment.

\section{Results}

Basic characteristics of study participants. Blood samples from patients with five types of liver disease were analyzed. The viral liver disease cohort included a total of 32 patients (13 females and 19 males; age, 18-75 years) who were infected with the hepatitis virus, mainly hepatitis B and hepatitis C virus. In the DILD cohort, there were a total of 39 patients (23 females and 16 males; age, 12-83 years) who had liver damage caused by adverse drug reactions. These drug reactions included those caused by herbals and antimicrobials such as amoxicillin-clavulanate, nitrofurantoin and sulfamethoxazole-trimethoprim, which of which can cause liver diseases (36,37). Additionally, 26 patients, 28 patients and 29 patients were respectively included into the alcoholic liver disease cohort (1 female and 25 males; age, 36-77 years), the PBC cohort (25 females and 3 males; age, 20-78 years) and the AIH cohort (23 females and 6 males; age, $16-76$ years) and 30 patients comprised the $\mathrm{HC}$ cohort (13 females and 17 males; age, 28-75 years).

The baseline characteristics of the participants in the present study are presented in Table I. Among the five cohorts, there were significant differences in sex, with more males exhibiting alcoholic liver disease and more females exhibiting PBC compared with those in the HC group. Laboratory parameters also significantly differed among the six cohorts. All patients with liver diseases exhibited higher AST, GGT and ALP levels but lower albumin levels compared those in the HC group. In the DILD group, patients exhibited higher ALT and bilirubin levels compared with those in the HC group. Data were presented as the median (interquartile range, 25th and 75th percentile). Bilirubin levels in the alcoholic liver disease group were demonstrated to be $\sim 7 \mathrm{X}$ higher compared with those in the HC group.

Serum IgG subclass levels among patients with different liver diseases. Serum concentrations of IgG subclasses are presented in Table II and Fig. 1. Levels of each IgG subclass and ratio of the $\mathrm{IgG}$ subclasses to total $\mathrm{IgG}$ significantly differed among the six cohorts in the present study. However, this was not observed for $\mathrm{IgG} 2 . \operatorname{IgG} 1$ levels in patients with $\mathrm{AIH}$ and the $\mathrm{IgG} 1 / \mathrm{IgG}$ ratio in patients with viral liver disease were significantly higher compared with those in the HC group. Additionally, higher levels of $\mathrm{IgG} 3$ and $\mathrm{IgG} 3 / \mathrm{IgG}$, and lower levels of $\mathrm{IgG} 4$ and $\mathrm{IgG} 4 / \mathrm{IgG}$ were detected in patients with PBC compared with those in the HC group. No notable in the distribution characteristics of IgG subclasses could be observed in the alcoholic liver disease and DILD cohorts in the present study. IgG1 levels were also significantly correlated with those of serum albumin in patients with AIH ( $\mathrm{r}=0.488), \mathrm{PBC}(\mathrm{r}=0.709)$, DILD $(\mathrm{r}=0.578)$, alcoholic liver disease $(\mathrm{r}=0.644)$ and viral liver disease $(r=0.534)$. The results also revealed that ALT, AST, GGT, ALP and bilirubin levels were not significantly associated with the levels of any of the IgG subclasses.

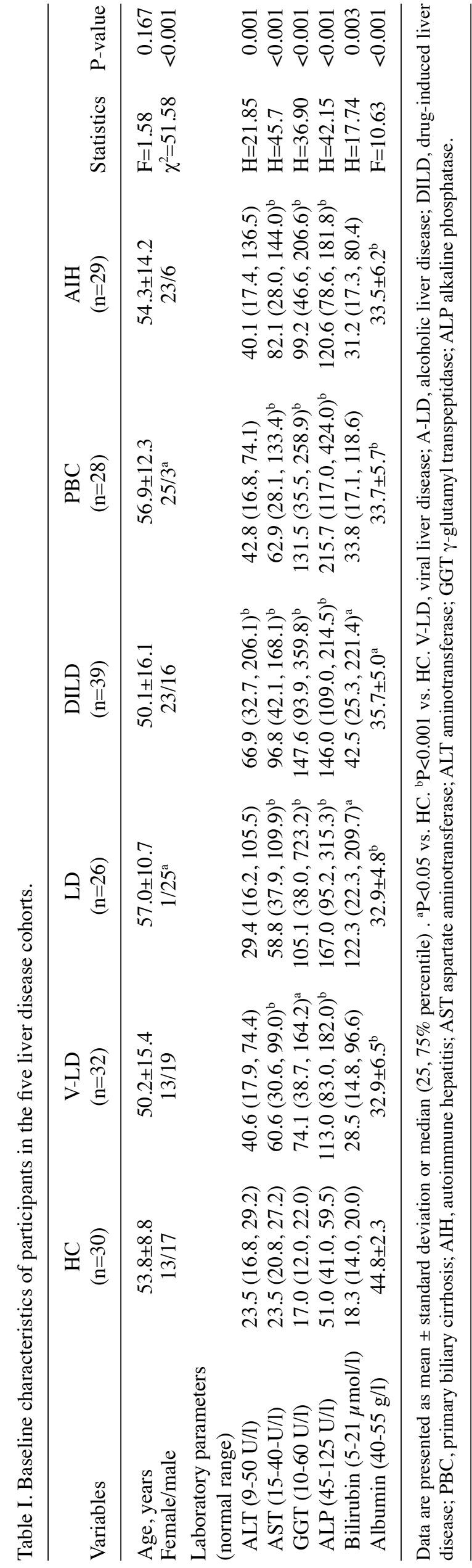


A
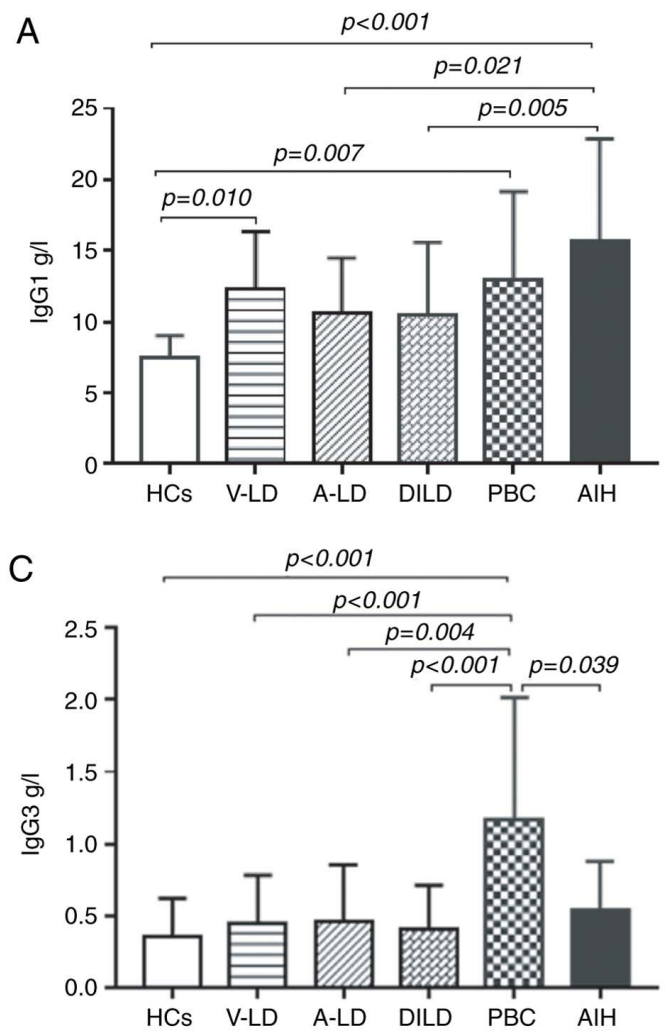

B

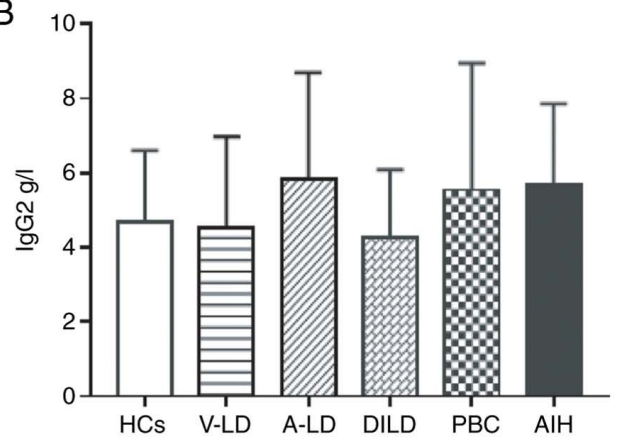

D

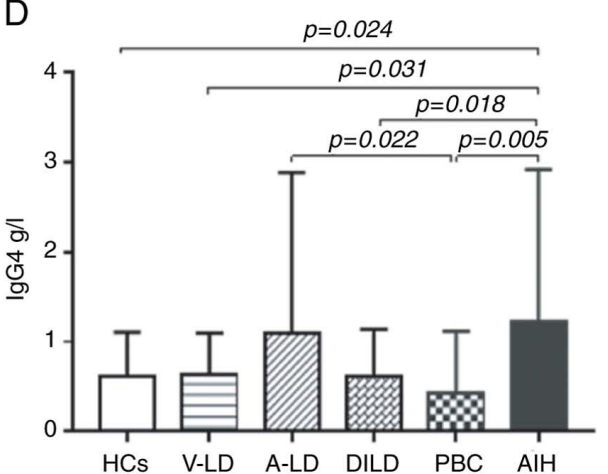

Figure 1. Serum IgG subclass levels among patients with different liver diseases. (A) IgG1 levels. Data are presented as the mean \pm standard deviation from three independent experiments. (B) IgG2, (C), IgG3 and (D) IgG4 levels. Data are presented as the median and 25th-75th percentiles from three independent experiments. Multiple comparison between the groups was performed using Scheffe post hoc test following Kruskal-Wallis tests. HCs, healthy controls; V-LD, viral liver disease; A-LD, alcoholic liver disease; DILD, drug-induced liver disease; PBC, primary biliary cirrhosis; AIH, autoimmune hepatitis.

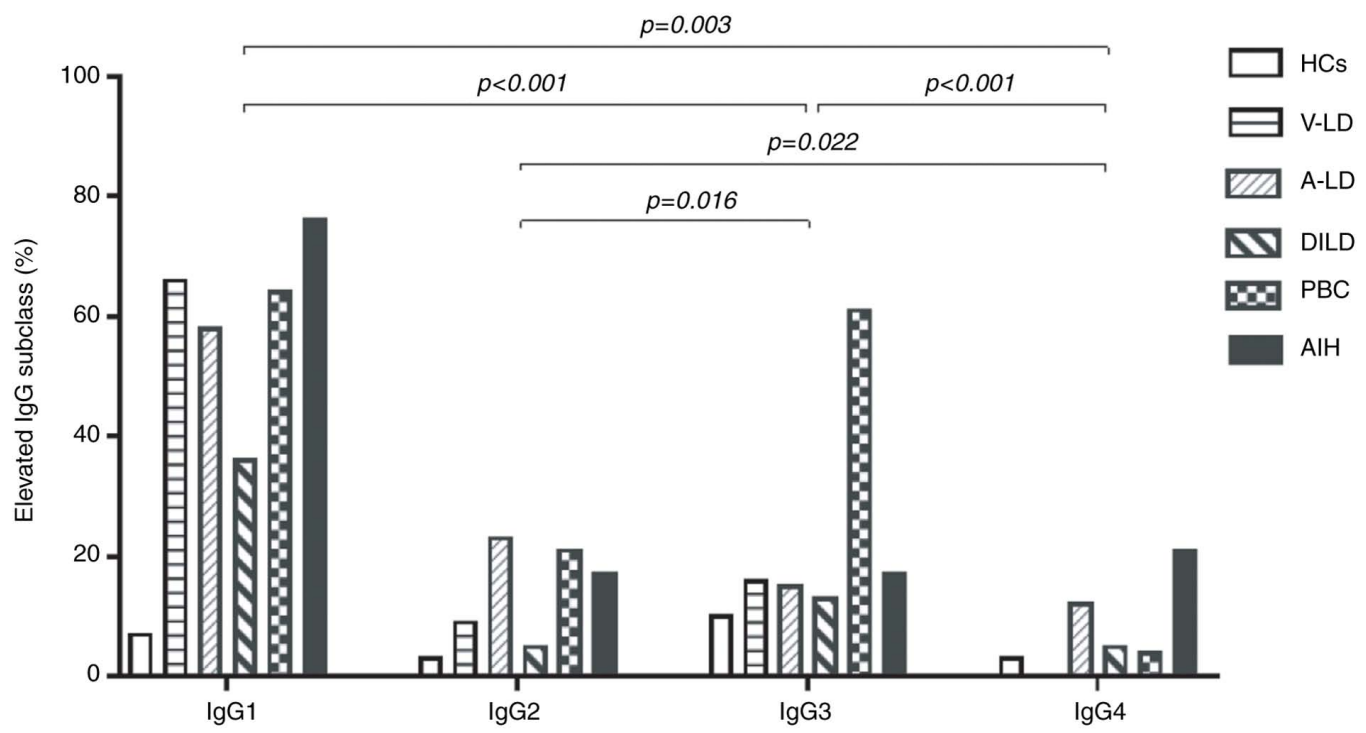

Figure 2. Elevated percentage of each IgG subclass among the different cohorts. All the statistical tests were performed using $\chi^{2}$ test. HCs, healthy controls; V-LD, viral liver disease; A-LD, alcoholic liver disease; DILD, drug-induced liver disease; PBC, primary biliary cirrhosis; AIH, autoimmune hepatitis.

IgG subclass levels in each patient cohort. The percentage of patients with PBC exhibiting elevated IgG3 levels was four times higher compared with that in the other groups (Fig. 2). The frequency of elevated IgG1 in patients with AIH was the highest of all cohorts, whilst the percentage of elevated IgG1 in patients with DILD was the lowest compared with that in the five disease groups. Compared with other IgG subclasses, the percentages of IgG1 and IgG4 elevations were the highest and lowest, respectively. In addition, the percentage of IgG1 elevation was markedly higher in all five cohorts compared with the other IgG subclasses.

Frequency of elevated $\operatorname{Ig} G$ subclasses in each group and the merged cohort. Overall, 68.2\% (105/154) participants in 


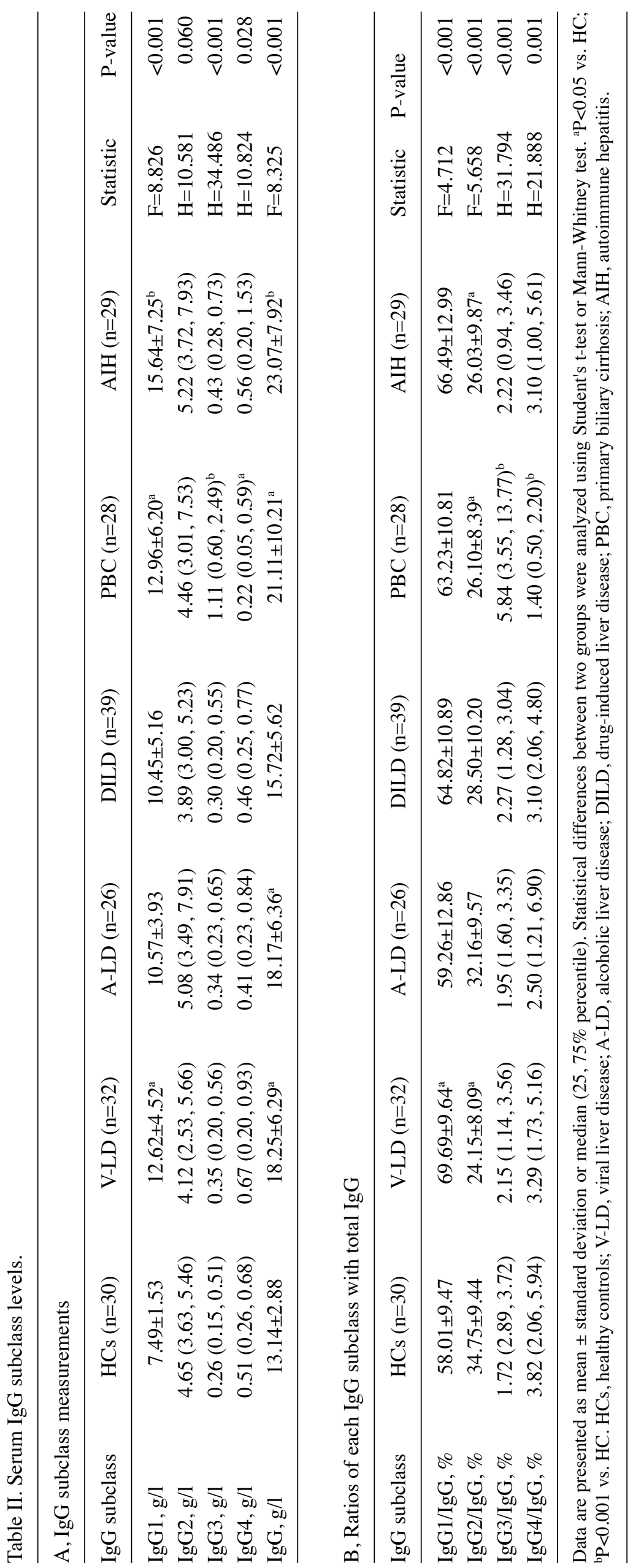


Table III. Frequency of elevated IgG subclasses in each group and all five cohorts combined.

\begin{tabular}{|c|c|c|c|c|c|}
\hline Patient group & $\begin{array}{l}\text { One type of increased } \\
\text { IgG subclass }{ }^{\mathrm{a}}(\mathrm{n}, \%)\end{array}$ & $\begin{array}{c}\text { Two types of } \\
\text { increased IgG } \\
\text { subclasses }^{\mathrm{b}}(\mathrm{n}, \%)\end{array}$ & $\begin{array}{l}\text { Three types of } \\
\text { increased IgG } \\
\text { subclasses }^{c}(n, \%)\end{array}$ & $\begin{array}{c}\operatorname{IgG} 1, \operatorname{IgG} 2, \\
\text { IgG3 and IgG4 } \\
\text { increased }^{\mathrm{d}}(\mathrm{n}, \%)\end{array}$ & $\begin{array}{c}\text { Only IgG4 } \\
\text { increased } \\
(\mathrm{n}, \%)\end{array}$ \\
\hline V-LD $(n=32)$ & $17(53.1)$ & $3(9.4)$ & $2(6.2)$ & $0(0)$ & $0(0)$ \\
\hline A-LD $(n=26)$ & $12(46.2)$ & $3(11.5)$ & $2(7.7)$ & $1(3.8)$ & $3(11.5)$ \\
\hline DILD $(n=39)$ & $11(28.2)$ & $6(15.4)$ & $0(0)$ & $0(0)$ & $2(5.1)$ \\
\hline $\operatorname{PBC}(n=28)$ & 8 (28.6) & $9(32.1)$ & $4(14.3)$ & $1(3.6)$ & $1(3.6)$ \\
\hline AIH $(n=29)$ & $18(62.1)$ & $5(17.2)$ & $2(6.9)$ & $1(3.4)$ & $6(20.7)$ \\
\hline $\begin{array}{l}\text { All five cohorts } \\
\text { combined }(n=154)\end{array}$ & $66(42.8)$ & $26(16.9)$ & $10(6.5)$ & $3(1.9)$ & $12(7.8)$ \\
\hline
\end{tabular}

${ }^{\mathrm{a} N u m b e r}$ of patients whose $\mathrm{IgG} 1, \mathrm{IgG} 2, \mathrm{IgG} 3$ or IgG4 was elevated. ${ }^{\mathrm{b}}$ Number of patients who exhibited two types of elevated IgG subclasses, specifically IgG1 and IgG2; IgG1 and IgG3; IgG1 and IgG4; IgG2 and IgG3; IgG2 and IgG4; or IgG3 and IgG4. 'Number of patients who exhibited three types of elevated IgG subclasses, specifically IgG1, IgG2 and IgG3; IgG1, IgG2 and IgG4; IgG1, IgG3 and IgG4; or IgG2, IgG3 and IgG4. ${ }^{\mathrm{d}} \mathrm{All}$ four types of IgG subclasses were elevated. The normal range for IgG levels in adults used in this analysis were: i) IgG1, 4.05-10.11 g/l; ii) IgG2, 1.69-7.86 g/l; iii) IgG3, 0.11-0.85 g/l; and iv) IgG4, 0.03-2.01 g/l. HCs, healthy controls; V-LD, viral liver disease; A-LD, alcoholic liver disease; DILD, drug-induced liver disease; PBC, primary biliary cirrhosis; AIH, autoimmune hepatitis.

Table IV. The titer of ANA and ASMA between PBC and AIH patients.

\begin{tabular}{|c|c|c|c|c|c|c|c|c|c|c|}
\hline \multirow[b]{2}{*}{ Group } & \multicolumn{4}{|c|}{ ANA titer } & \multicolumn{4}{|c|}{ ASMA titer } & \multicolumn{2}{|c|}{ Positive rates $(\%, \mathrm{n})$} \\
\hline & Negative & $1: 100$ & $1: 320$ & $1: 1,000$ & Negative & $1: 100$ & $1: 320$ & $1: 1,000$ & ANA & ASMA \\
\hline $\operatorname{PBC}(n=26)^{a}$ & 5 & 2 & 1 & 18 & 26 & 0 & 0 & 0 & $80.8 \%(21 / 26)$ & $0 \%(0 / 26)$ \\
\hline $\operatorname{AIH}(n=28)^{b}$ & 3 & 3 & 6 & 16 & 19 & 3 & 4 & 2 & $89.3 \%(25 / 28)$ & $32.1 \%(9 / 28)$ \\
\hline
\end{tabular}

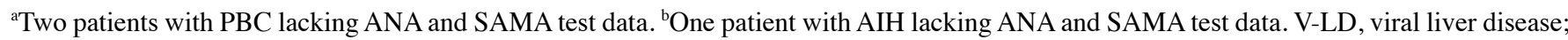
A-LD, alcoholic liver disease; DILD, drug-induced liver disease; PBC, primary biliary cirrhosis; AIH, autoimmune hepatitis.

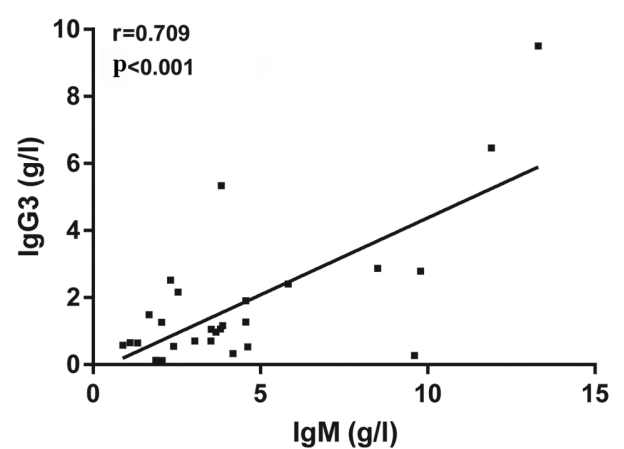

Figure 3. Correlation between $\operatorname{IgG} 3$ and IgM concentrations in patients with PBC.

the merged cohort (five cohorts combined) had $\geq$ one type of elevated IgG subclass (Table III). The most frequently observed increase was one type of increased IgG subclass, whilst four types of elevated IgG subclasses was the least common. In $25.3 \%$ (39/154) patients, $\geq$ two IgG subclasses were simultaneously increased.

Relationship between IgG3 and IgM concentrations in patients with PBC. IgA levels in patients with $\mathrm{PBC}$ were found to be $3.65(1.78,5.82) \mathrm{g} / \mathrm{l}$ and $3.06(1.53,6.30) \mathrm{g} / \mathrm{l}$ in patients with AIH. Data were presented as the median (interquartile range, 25 and 75 th percentile). IgA did not significantly differ between patients with PBC and $\mathrm{AIH}$. IgM levels in patients with PBC were $3.60(2.12,4.60) \mathrm{g} / 1$ and $1.80(1.0,3.0) \mathrm{g} / \mathrm{l}$ in patients with with AIH. IgM levels in patients with PBC were significantly higher compared with that in patients with AIH. Pearson's correlation coefficient analysis was subsequently used to evaluate the relationship between $\operatorname{IgG} 3$ and $\operatorname{IgM}$ concentrations in patients with $\mathrm{PBC}$. The results revealed that IgG3 levels were significantly correlated with those of serum IgM in patients with PBC (Fig. 3). These results suggested that IgG3 and IgM may serve synergistic effects in patients with PBC.

Relationship between concentrations of IgG3 and AMA-M2 in patients with $P B C$. $\mathrm{PBC}$ is an autoimmune disease of the liver characterized by the presence of AMA in $90-95 \%$ of patients (38). Serum AMA, particularly that of the AMA-M2 subtype, is regarded to be one of the most specific and acceptable diagnostic indicators of PBC (39). In the present study, IgG3 levels were not found to be significantly associated with AMA-M2 titers in patients with PBC (Fig. 4). A total of $94.1 \%$ patients with PBC demonstrated medium to 


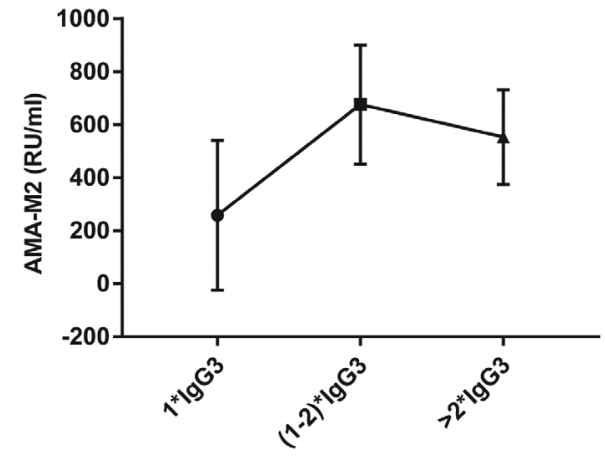

Figure 4. Relationship between concentrations of IgG3 and AMA-M2 in patients with $\mathrm{PBC}$. Each datapoint was presented as the mean \pm standard deviation. AMA-M2, anti-mitochondrial M2; $1 * \operatorname{IgG} 3$, normal reference range values of $\operatorname{IgG} 3 ;(1-2) * \operatorname{IgG} 3,(1-2)$ times of reference range of $\mathrm{IgG} 3$; $>2 * \operatorname{IgG} 3, \mathrm{IgG} 3$ levels $>2 \mathrm{X}$ upper limit of normal range.

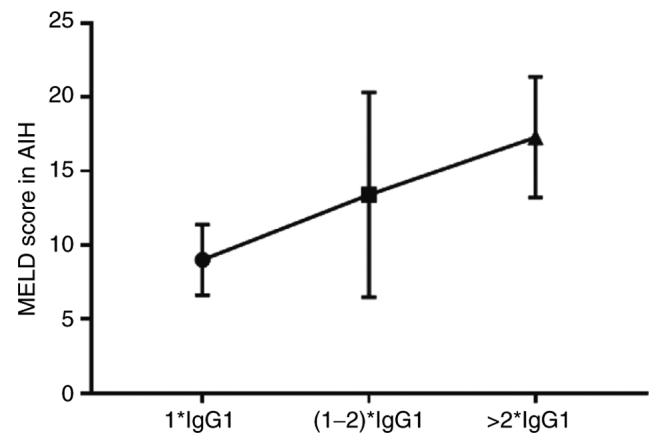

Figure 5. Association between IgG1 subclass concentration and MELD liver damage score in patients with AIH. Each datapoint was presented as the mean \pm standard deviation. MELD, model for end-stage liver disease; $\mathrm{AIH}$, autoimmune hepatitis; $1 * \mathrm{IgG} 1$, normal reference range values of $\mathrm{IgG1}$; (1-2)*IgG1, 1-2 times of reference ranges of IgG1; $>2 * \operatorname{IgG} 1$, IgG1 levels $>2 \mathrm{X}$ upper limit of normal.

high AMA-M2 titers (AMA-M2 $\geq 200 \mathrm{RU} / \mathrm{ml}$ ) when IgG3 was elevated. When elevated IgG3 was $1-2 \mathrm{X}$ higher than the reference interval, AMA-M2 titers were also higher compared with two groups of $1 \mathrm{X}$ and $>2 \mathrm{X}$ reference interval of IgG3 (Fig. 4). The positive rate of the ANA autoantibody in patients with PBC was $80.8 \%(21 / 26)$ and $89.3 \%(25 / 28)$ in patients with AIH (Table IV), due to two patients with PBC and one patient with AIH lacking ANA test data. ANA titers did not significantly differ between patients with PBC and AIH. ASMA could not be detected in patients in the PBC group whereas the positive rate of ASMA in patients with AIH was $32.1 \%(9 / 28)$ (Table IV). The incidence of ASMA positivity in patients with AIH were significantly higher compared with those in the PBC cohort $\left(\chi^{2}=10.03\right.$; $\mathrm{P}=0.002)$.

Association between IgG subclass concentration and liver damage in patients with AIH. MELD scores are used to calculate the degree of liver damage, such that the higher the MELD score, the greater the liver damage (40). Patients with AIH demonstrated higher MELD scores (13.28 \pm 6.10$)$ compared with those in the PBC cohort $(12.63 \pm 5.27)$, but statistical significance was not observed. In patients with AIH, IgG1 levels did not significantly associate with the MELD
Table V. Frequency of elevated serum IgG4 levels in patients.

\begin{tabular}{lccc}
\hline Groups & Cases & $\mathrm{N}(\mathrm{IgG} 4>135 \mathrm{mg} / \mathrm{dl}, \%)$ & P-value $^{\mathrm{a}}$ \\
\hline V-LD & 32 & $3(9.4)$ & - \\
A-LD & 26 & $4(15.4)$ & 0.222 \\
DILD & 39 & $3(7.7)$ & 0.809 \\
PBC & 28 & $1(3.6)$ & 0.165 \\
AIH & 29 & $6(20.7)$ & 0.079
\end{tabular}

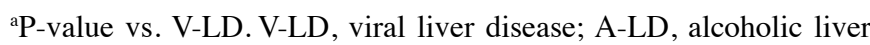
disease; DILD, drug-induced liver disease; PBC, primary biliary cirrhosis; AIH, autoimmune hepatitis.

scores. However, within the same cohort, MELD scores were the highest in the cohort exhibiting IgG1 levels $>$ two times higher than the reference intervals (Fig. 5). IgG1 levels and MELD scores may therefore share a synergistic relationship.

Frequency of elevated serum IgG4 levels in patients. Serum IgG4 levels $>135 \mathrm{mg} / \mathrm{dl}$ are considered to be diagnostic criteria for IgG4-RD (18). The number and frequency of patients with serum IgG4 levels $>135 \mathrm{mg} / \mathrm{dl}$ were therefore calculated among the five cohorts. Among the 154 patients, only 17 (11.0\%) had serum IgG4 levels $>135 \mathrm{mg} / \mathrm{dl}$ (Table V). In addition, no significant difference in the frequency of elevated serum IgG4 levels was identified between patients with viral liver disease and patients without non-viral liver disease (Table V).

\section{Discussion}

Liver diseases can be caused by a variety of factors, including genetic predisposition, infection, autoimmunity or metabolism, which renders diagnosis challenging (4). In the present retrospective study, serum IgG subclass levels were analyzed in patients with five common liver diseases to further their understanding and the development of efficient novel early diagnosis strategies. The results revealed that certain serum IgG subclass levels were selectively increased or decreased depending of the type of disease.

Previous studies have demonstrated that serum IgG3 levels are elevated patients with PBC $(41,42)$. However, little is known about IgG subclasses in patients with AIH. The results of the present study suggested that there were higher levels of IgG3 and $\mathrm{IgG} 3 / \mathrm{IgG}$ but lower levels of $\mathrm{IgG} 4$ and $\mathrm{IgG} / \mathrm{IgG}$ in patients with $\mathrm{PBC}$ compared with the $\mathrm{HC}$ group, further validating the results of aforementioned studies. Additionally, compared with those in the $\mathrm{HC}$ group, IgG1 levels were significantly higher in patients with AIH. PBC and AIH are autoimmune diseases. The results of the present study indicated that IgG subclasses may serve a role in the differential diagnosis of patients with PBC and AIH. The four subclasses of IgG differ in their constant regions, particularly in their hinges and $\mathrm{CH} 2$ domains. IgG1 has the highest Fc $\gamma \mathrm{R}$ binding affinity, followed by $\operatorname{IgG} 3, \operatorname{IgG} 2$ and IgG4 in that order (43). When antigens enter the body, their chemical compositions stimulate an immune reaction, resulting in differential patterns of class switching. For different antigens, such as adhesion protein desmoglein 3 , 
interleukin (IL)-4 and IL-10, class switching tends to occur from IgG1 or IgG3 to IgG4 $(44,45)$.

A previous study revealed that alcoholic liver disease was associated with low serum concentrations of IgG and reduced IgG following alcohol administration in mice (46). However, the results of the present study demonstrated that IgG levels were elevated, which was not consistent with observations from this previous study. This may be due to different effects exerted by alcohol in murine and human systems. Alonso et al (46) previously injected alcohol into mice and measured $\mathrm{IgG}$ concentrations after 4 weeks and found that alcohol administration appeared to decrease $\operatorname{IgG}$ subclass concentrations. Patients with alcoholic liver disease frequently have a long history of drinking (47). It therefore may take some time for the IgG subclasses to be produced in patients with alcoholic liver disease. Additionally, Alonso's research objects were mice, the physiological functions of which were different from those of humans. As a result, further research is required to assess these affects in humans.

Previous studies have demonstrated that when compared with $\mathrm{HCs}$, serum IgG1 and IgG3 levels were higher in virus-infected individuals (48). In the present study, IgG1/IgG levels were significantly increased in viral liver disease group when compared with HCs. IgG3 was also higher compared with that of HCs, but without significance. IgG serves a pivotal role in viral neutralization (49). Antigens can trigger B-cells directly, leading to a plethora of secondary signals that regulates cell differentiation and subsequent antibody production $(50,51)$. The induction of specific IgG1 and IgG3 antibodies is therefore more likely to achieve the clearance of HBV and HCV (49).

The results of the present study revealed that the expression of IgG3 in patients with PBC was significantly correlated with the levels of serum IgM. Furthermore, $94.1 \%$ patients with PBC demonstrated medium to high AMA-M2 titers (AMA-M2 $\geq 200$ RU/ml) when IgG3 was elevated. Serum IgM and AMA-M2 levels are two of the most important and specific diagnostic indicators of PBC (52). IgG3, IgM and AMA-M2 may have synergistic effects in $\mathrm{PBC}$, which may be involved in the pathogenesis of PBC. In addition, since AMA-M2 is primarily of the IgG-AMA subclass (52) and that currently available commercial kits are customized for IgG-AMA detection, measurement of AMA and IgG3 levels may be more accurate than radioimmunodiffusion in diagnosing PBC.

The MELD score was designed to calculate the degree of liver damage, where higher MELD score implies greater damage to the liver (40). In the present study, it was demonstrated that IgG1 levels and MELD scores may have a synergistic relationship in patients with AIH. Different antigens can stimulate an IgG1 response (51). Therefore, excessive IgG1 levels are more likely to harm the liver and cause autoimmune liver disease $(53,54)$.

IgG4-RD is a systemic disease involving a number of organs, including the pancreas, lacrimal glands, lungs, liver and kidney (55). Two types of liver disease involvement have been reported in relation to IgG4-RD: IgG4-related AIH and IgG4-hepatopathy (55). Certain common liver diseases have been reported to share similarities with IgG4-RD. IgG4-related AIH is clinicopathologically similar to that of AIH, except for the elevated serum IgG4 levels and heavy infiltration of
IgG4-positive plasma cells in the liver tissue (56). In addition, IgG4-related AIH can be hard to diagnose as well-known IgG4-RD(s) (55). Yamamoto et al (28) revealed that except for IgG4-RD, increased IgG4 levels were observed several clinical cases, such as Churg-Strauss syndrome, multicentric Castleman's disease, eosinophilic disorders, and some patients with rheumatoid arthritis, systemic sclerosis, chronic hepatitis, and liver cirrhosis. Additionally, Ebbo et al (57) reported that $13.6 \%$ patients whose serum IgG4 levels were $>$ the cutoff value of $135 \mathrm{mg} / \mathrm{dl}$ were diagnosed with IgG4-RD. In the present study, only $11 \%$ patients exhibited serum IgG4 levels $>135 \mathrm{mg} / \mathrm{dl}$, which was lower compared with that of previous studies. The results suggested that serum IgG4 levels in the five patient cohorts of the present study were different from those with IgG4-RD in the previous studies aforementioned. These data may prove to be useful for differential diagnosis between IgG4-RD and five liver disease cohorts, although they share similarities of elevated serum IgG4 levels in pathogenic processes. Additionally, the present study suggested that IgG4 and other IgG subclasses may be used to distinguish liver diseases from IgG4-RD.

The present study has several limitations. Firstly, since this was an observational study, the effect of IgG4 on the clinical manifestation of patients was not determined. Therefore, a follow-up process should be performed in future studies. Secondly, different laboratories could have slightly different definitions for elevated IgG4. Finally, the number of samples in the present study was relatively small and the present study lacked patients at different stages of liver disease.

In conclusion, different liver diseases were found to be associated with different serum IgG subclass distributions. The present study suggested that IgG subclasses may serve as biomarkers for the early diagnosis of liver diseases. However, further studies on serum IgG subclass distributions may further elucidate the immunopathogenesis of liver disease.

\section{Acknowledgements}

Not applicable.

\section{Funding}

The present study was supported by the Beijing Natural Science Foundation (grant nos. 7191004 and 7202069), the Beijing Municipal Science \& Technology Commission (grant no. Z171100001017078), the Beijing municipal administration of hospitals (grant nos. DFL20181701 and ZYLX201711) and the Beijing Key Laboratory (grant no. BZ0373). The Capital health research and development of special (grant no. Capital development 2020-2-1153).

\section{Availability of data and materials}

The datasets used and/or analyzed during the current study are available from the corresponding author on reasonable request.

\section{Authors' contributions}

WZ drafted the manuscript. WZ and FJ collected the data. JS, YW, YJ, QG and JL performed the experiments. WZ and YZ 
performed the statistical analysis and participated in the study design. WZ and YZ participated in the acquisition, analysis or interpretation of the data. All authors read and approved the final manuscript.

\section{Ethics approval and consent to participate}

The present study was approved by the Ethics Committee of Beijing YouAn Hospital, Capital Medical University (Beijing, China).

\section{Patient consent for publication}

Not applicable.

\section{Competing interests}

All authors declare that they have no competing interests.

\section{References}

1. Asrani SK, Devarbhavi H, Eaton J and Kamath PS: Burden of liver diseases in the world. J Hepatol 70: 151-171, 2019.

2. Rowe IA: Lessons from epidemiology: The burden of liver disease. Dig Dis 35: 304-309, 2017.

3. GBD 2013 DALYs and HALE Collaborators; Murray CJ, Barber RM, Foreman KJ, Abbasoglu Ozgoren A, Abd-Allah F, Abera SF, Aboyans V, Abraham JP, Abubakar I, et al: Global, regional, and national disability-adjusted life years (DALYs) for 306 diseases and injuries and healthy life expectancy (HALE) for 188 countries, 1990-2013: Quantifying the epidemiological transition. Lancet 386: 2145-2191, 2015.

4. Wang FS, Fan JG, Zhang Z, Gao B and Wang HY: The global burden of liver disease: The major impact of China. Hepatology 60: 2099-2108, 2014.

5. Carbone M and Neuberger JM: Autoimmune liver disease, autoimmunity and liver transplantation. J Hepatol 60: 210-223, 2014

6. Kapsogeorgou EK and Tzioufas AG: Autoantibodies in autoimmune diseases: Clinical and critical evaluation. Isr Med Assoc J 18: 519-524, 2016.

7. Dusseaux M, Masse-Ranson G, Darche S, Ahodantin J, Li Y, Fiquet O, Beaumont E, Moreau P, Riviere L, Neuveut C, et al: Viral load affects the immune response to HBV in mice with humanized immune system and liver. Gastroenterology 153 1647-1661.e9, 2017.

8. Berry AA, Gottlieb ER, Kouriba B, Diarra I, Thera MA, Dutta S, Coulibaly D, Ouattara A, Niangaly A, Kone AK, et al Immunoglobulin $\mathrm{G}$ subclass and antibody avidity responses in Malian children immunized with Plasmodium falciparum apical membrane antigen 1 vaccine candidate FMP2.1/AS02 ${ }_{\mathrm{A}}$. Malar J 18: 13, 2019.

9. Vidarsson G, Dekkers G and Rispens T: IgG subclasses and allotypes: From structure to effector functions. Front Immunol 5 : $520,2014$.

10. Valenzuela NM and Schaub S: The Biology of IgG subclasses and their clinical relevance to transplantation. Transplantation 102 (Suppl 1): S7-S13, 2018.

11. Jansen A, Mandić AD, Bennek E, Frehn L, Verdier J, Tebrügge I, Lutz H, Streetz K, Trautwein C and Sellge G: Anti-food and anti-microbial $\mathrm{IgG}$ subclass antibodies in inflammatory bowel disease. Scand J Gastroenterol 51: 1453-1461, 2016.

12. Khokar A and Gupta S: Clinical and immunological features of 78 adult patients with primary selective IgG subclass deficiencies. Arch Immunol Ther Exp (Warsz) 67: 325-334, 2019.

13. Shen H, Zhang M, Kaita K, Minuk GY, Rempel J and Gong Y: Expression of Fc fragment receptors of immunoglobulin $\mathrm{G}$ $(\mathrm{Fc} \gamma \mathrm{Rs})$ in rat hepatic stellate cells. Dig Dis Sci 50: 181-187, 2005.

14. Pressler T, Mansa B, Pedersen SS, Espersen F, Høiby N and Koch C: Methodologic problems in establishing normal values for IgG subclass concentrations in a pediatric population; comparison of radial immunodiffusion and ELISA methods. Allergy 49: 772-777, 1994.
15. van der Gugten G, DeMarco ML, Chen LYC, Chin A, Carruthers M, Holmes DT and Mattman A: Resolution of spurious immunonephelometric IgG subclass measurement discrepancies by LC-MS/MS. Clin Chem 64: 735-742, 2018.

16. Brito-Zeron P, Ramos-Casals M, Bosch X and Stone JH: The clinical spectrum of IgG4-related disease. Autoimmun Rev 13: 1203-1210, 2014.

17. Okazaki $\mathrm{K}$ and Umehara $\mathrm{H}$ : Current concept of IgG4-related disease. Curr Top Microbiol Immunol 401: 1-17, 2017.

18. Khosroshahi A, Wallace ZS, Crowe JL, Akamizu T, Azumi A, Carruthers MN, Chari ST, Della-Torre E, Frulloni L, Goto $\mathrm{H}$, et al: International consensus guidance statement on the management and treatment of IgG4-related disease. Arthritis Rheumatol 67: 1688-1699, 2015.

19. Brito-Zerón P, Bosch X, Ramos-Casals $M$ and Stone JH: IgG4-related disease: Advances in the diagnosis and treatment. Best Pract Res Clin Rheumatol 30: 261-278, 2016.

20. Kamisawa T, Zen Y, Pillai S and Stone JH: IgG4-related disease. Lancet 385: 1460-1471, 2015.

21. Culver EL and Chapman RW: IgG4-related hepatobiliary disease: An overview. Nat Rev Gastroenterol Hepatol 13: 601-612, 2016.

22. Westra J, van Assen S, Wilting KR, Land J, Horst G, de Haan A and Bijl M: Rituximab impairs immunoglobulin (Ig)M and IgG (subclass) responses after influenza vaccination in rheumatoid arthritis patients. Clin Exp Immunol 178: 40-47, 2014.

23. Huang CC, Lehman A, Albawardi A, Satoskar A, Brodsky S, Nadasdy G, Hebert L, Rovin B and Nadasdy T: IgG subclass staining in renal biopsies with membranous glomerulonephritis indicates subclass switch during disease progression. Mod Pathol 26: 799-805, 2013.

24. Sadanand S, Das J, Chung AW, Schoen MK, Lane S, Suscovich TJ, Streeck H, Smith DM, Little SJ, Lauffenburger DA, et al: Temporal variation in HIV-specific IgG subclass antibodies during acute infection differentiates spontaneous controllers from chronic progressors. AIDS 32: 443-450, 2018.

25. Zhang YL, Wang ZF and Chen N: Expression of serum IgG4 in patients with different diseases. Beijing Da Xue Xue Bao Yi Xue Ban 49: 961-964, 2017 (In Chinese)

26. Zuo Y, Evangelista F, Culton D, Guilabert A, Lin L, Li N, Diaz L and Liu Z: IgG4 autoantibodies are inhibitory in the autoimmune disease bullous pemphigoid. J Autoimmun 73: 111-119, 2016.

27. Liu Y and Li J: Preferentially immunoglobulin (IgG) subclasses production in primary Sjogren's syndrome patients. Clin Chem Lab Med 50: 345-349, 2011.

28. Yamamoto M, Tabeya T, Naishiro Y, Yajima H, Ishigami K, Shimizu Y, Obara M, Suzuki C, Yamashita K, Yamamoto H, et al: Value of serum IgG4 in the diagnosis of IgG4-related disease and in differentiation from rheumatic diseases and other diseases. Mod Rheumatol 22: 419-425, 2012.

29. Chinese Society of Hepatology, Chinese Medical Association; Chinese Society of Infectious Diseases, Chinese Medical Association; Hou JL and Lai W: The guideline of prevention and treatment for chronic hepatitis B: A 2015 update. Zhonghua Gan Zang Bing Za Zhi 23: 888-905, 2015 (In Chinese).

30. Chinese Society of Hepatology, Chinese Medical Association; Wei L; Chinese Society of Infectious Diseases, Chinese Medical Association and Hou JL: The guideline of prevention and treatment for hepatitis C: A 2015 update. Zhonghua Gan Zang Bing Za Zhi 23: 906-923, 2015 (In Chinese).

31. O'Shea RS, Dasarathy S and McCullough AJ: Alcoholic liver disease. Am J Gastroenterol 105: 14-32, 2010.

32. Danan G and Teschke R: Roussel Uclaf causality assessment method for drug-induced liver injury: Present and future. Front Pharmacol 10: 853, 2019.

33. Lindor KD, Bowlus CL, Boyer J, Levy C and Mayo M: Primary biliary cholangitis: 2018 practice guidance from the american association for the study of liver diseases. Hepatology 69: 394-419, 2019.

34. Chinese Society of Hepatology, Chinese Society of Gastroenterology \& Chinese Society of Infectious Diseases: Chinese consensus on the diagnosis and management of autoimmune hepatitis (2015). J Dig Dis 18: 247-264, 2017.

35. Kim S, Zerillo J, Tabrizian P, Wax D, Lin HM, Evans A, Florman S and DeMaria S Jr: Postoperative meld-lactate and isolated lactate values as outcome predictors following orthotopic liver transplantation. Shock 48: 36-42, 2017.

36. Fisher K, Vuppalanchi R and Saxena R: Drug-induced liver injury. Arch Pathol Lab Med 139: 876-887, 2015. 
37. Kleiner DE, Chalasani NP, Lee WM, Fontana RJ, Bonkovsky HL, Watkins PB, Hayashi PH, Davern TJ, Navarro V, Reddy R, et al: Hepatic histological findings in suspected drug-induced liver injury: Systematic evaluation and clinical associations. Hepatology 59: 661-670, 2014.

38. de Liso F, Matinato C, Ronchi M and Maiavacca R: The diagnostic accuracy of biomarkers for diagnosis of primary biliary cholangitis (PBC) in anti-mitochondrial antibody (AMA)-negative PBC patients: A review of literature. Clin Chem Lab Med 56: 25-31, 2017.

39. Huang YQ: Recent advances in the diagnosis and treatment of primary biliary cholangitis. World J Hepatol 8: 1419-1441, 2016.

40. Lebray P and Varnous S: Combined heart and liver transplantation: State of knowledge and outlooks. Clin Res Hepatol Gastroenterol 43: 123-130, 2019.

41. Rigopoulou EI, Davies ET, Bogdanos DP, Liaskos C, Mytilinaiou M, Koukoulis GK, Dalekos GN and Vergani D Antimitochondrial antibodies of immunoglobulin G3 subclass are associated with a more severe disease course in primary biliary cirrhosis. Liver Int 27: 1226-1231, 2007.

42. Zhang H, Li P, Wu D, Xu D, Hou Y, Wang Q, Li M, Li Y, Zeng X, Zhang F and Shi Q: Serum IgG subclasses in autoimmune diseases. Medicine (Baltimore) 94: e387, 2015.

43. Yu J, Song Y and Tian W: How to select IgG subclasses in developing anti-tumor therapeutic antibodies. J Hematol Oncol 13: 45, 2020.

44. Ellebrecht CT, Mukherjee EM, Zheng Q, Choi EJ, Reddy SG, Mao X and Payne AS: Autoreactive IgG and IgA B cells evolve through distinct subclass switch pathways in the autoimmune disease pemphigus vulgaris. Cell Rep 24: 2370-2380, 2018.

45. Nualnoi T, Kirosingh A, Basallo K, Hau D, GatesHollingsworth MA, Thorkildson P, Crump RB, Reed DE, Pandit $S$ and AuCoin DP: Immunoglobulin G subclass switching impacts sensitivity of an immunoassay targeting Francisella tularensis lipopolysaccharide. PLoS One 13: e0195308, 2018

46. Alonso M, Gomez-Rial J, Gude F, Vidal C and Gonzalez-Quintela A: Influence of experimental alcohol administration on serum immunoglobulin levels: Contrasting effects on IgE and other immunoglobulin classes. Int J Immunopathol Pharmacol 25: 645-655, 2012.

47. Mathurin $P$ and Bataller R: Trends in the management and burden of alcoholic liver disease. J Hepatol 62 (1 Suppl): S38-S46, 2015.

48. Walker MR, Eltahla AA, Mina MM, Li H, Lloyd AR and Bull RA: Envelope-specific IgG3 and IgG1 responses are associated with clearance of acute hepatitis $\mathrm{C}$ virus infection. Viruses 12: 75, 2020.
49. Jin J, Xu H, Wu R, Gao N, Wu N, Li S and Niu J: Identification of key genes and pathways associated with different immune statuses of hepatitis B virus infection. J Cell Mol Med 23: 7474-7489, 2019.

50. Pone EJ, Zhang J, Mai T, White CA, Li G, Sakakura JK, Patel PJ, Al-Qahtani A, Zan H, Xu Z and Casali P: BCR-signalling synergizes with TLR-signalling for induction of AID and immunoglobulin class-switching through the non-canonical NF-kappaB pathway. Nat Commun 3: 767, 2012.

51. Giuntini S, Granoff DM, Beernink PT, Ihle O, Bratlie D and Michaelsen TE: Human IgG1, IgG3, and IgG3 hinge-truncated mutants show different protection capabilities against meningococci depending on the target antigen and epitope specificity. Clin Vaccine Immunol 23: 698-706, 2016.

52. Tang L, Zhong R, He X, Wang W, Liu J, Zhu Y, Li Y and Hou J: Evidence for the association between IgG-antimitochondrial antibody and biochemical response to ursodeoxycholic acid treatment in primary biliary cholangitis. J Gastroenterol Hepatol 32: 659-666, 2017.

53. Behairy OG, Behiry EG, El Defrawy MS and El Adly AN: Diagnostic value of soluble programmed cell death protein-1 in type-1 autoimmune hepatitis in Egyptian children. Scand J Clin Lab Invest 80: 59-65, 2020.

54. Than NN, Ching DK, Hodson J, McDowell P, Mann J, Gupta R, Salazar E, Ngu JH and Oo YH: Difference in clinical presentation, immunology profile and treatment response of type 1 autoimmune hepatitis between United Kingdom and Singapore patients. Hepatol Int 10: 673-679, 2016.

55. Nakanuma Y, Ishizu Y, Zen Y, Harada K and Umemura T: Histopathology of IgG4-related autoimmune hepatitis and IgG4-related hepatopathy in IgG4-related disease. Semin Liver Dis 36: 229-241, 2016.

56. Lee HE and Zhang L: Immunoglobulin G4-related hepatobiliary disease. Semin Diagn Pathol 36: 423-433, 2019.

57. Ebbo M, Grados A, Bernit E, Vély F, Boucraut J, Harlé JR, Daniel L and Schleinitz N: Pathologies associated with serum IgG4 elevation. Int J Rheumatol 2012: 602809, 2012.

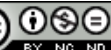

This work is licensed under a Creative Commons Attribution-NonCommercial-NoDerivatives 4.0 International (CC BY-NC-ND 4.0) License. 